MATHEMATICS OF COMPUTATION

Volume 76, Number 258, April 2007, Pages 615-629

S 0025-5718(06)01917-X

Article electronically published on November 27, 2006

\title{
AN OPTIMAL ADAPTIVE WAVELET METHOD WITHOUT COARSENING OF THE ITERANDS
}

\author{
TSOGTGEREL GANTUMUR, HELMUT HARBRECHT, AND ROB STEVENSON
}

\begin{abstract}
In this paper, an adaptive wavelet method for solving linear operator equations is constructed that is a modification of the method from [Math. Comp, 70 (2001), pp. 27-75] by Cohen, Dahmen and DeVore, in the sense that there is no recurrent coarsening of the iterands. Despite this, it will be shown that the method has optimal computational complexity. Numerical results for a simple model problem indicate that the new method is more efficient than an existing alternative adaptive wavelet method.
\end{abstract}

\section{Preliminaries}

For some boundedly invertible linear operator $A: H \rightarrow H^{\prime}$, where $H$ is some separable Hilbert space with dual $H^{\prime}$, and some $f \in H^{\prime}$, we consider the problem of finding $u \in H$ such that

$$
A u=f .
$$

As typical examples, we think of linear differential or integral equations of some order $2 t$ in variational form. Furthermore, although systems of such equations also fit into the framework, usually we think of scalar equations. So typically $H$ is a Sobolev space $H^{t}$, possibly incorporating essential boundary conditions, on an $n$-dimensional underlying domain or manifold.

We assume that we have a Riesz basis $\Psi=\left\{\psi_{\lambda}: \lambda \in \nabla\right\}$ for $H^{t}$ available, where $\nabla$ is some infinite countable index set. Formally viewing this basis as a column vector, by writing $u=\mathbf{u}^{T} \Psi$ the above problem is equivalent to finding $\mathbf{u} \in \ell_{2}=\ell_{2}(\nabla)$ satisfying the infinite matrix-vector system

$$
\mathbf{A u}=\mathbf{f},
$$

where the stiffness matrix $\mathbf{A}:=\langle\Psi, A \Psi\rangle: \ell_{2} \rightarrow \ell_{2}$ is boundedly invertible and $\mathbf{f}:=\langle\Psi, f\rangle \in \ell_{2}$. Here $\langle\cdot, \cdot\rangle$ denotes the duality product on $\left(H^{t}, H^{-t}\right)$. In the following, we will also use $\langle\cdot, \cdot\rangle$ to denote $\langle\cdot, \cdot\rangle_{\ell_{2}}$, and use $\|\cdot\|$ to denote $\|\cdot\|_{\ell_{2}}$ as well as $\|\cdot\|_{\ell_{2} \rightarrow \ell_{2}}$. Throughout this paper, $\mathbf{u}$ and $\mathbf{f}$ will always denote the solution and right-hand side of this equation, respectively.

Let us denote by $\mathbf{u}_{N}$ a best $N$-term approximation for $\mathbf{u}$, i.e., a vector with at most $N$ nonzero coefficients that has distance to $\mathbf{u}$ not larger than that of any vector with a support of that size. Note that $\left\|u-\mathbf{u}_{N}^{T} \Psi\right\|_{H^{t}} \approx\left\|\mathbf{u}-\mathbf{u}_{N}\right\|$. We will

Received by the editor March 22, 2005 and, in revised form, January 25, 2006.

2000 Mathematics Subject Classification. Primary 41A25, 41A46, 65F10, 65T60.

Key words and phrases. Adaptive methods, operator equations, wavelets, optimal computational complexity, best $N$-term approximation.

This work was supported by the Netherlands Organization for Scientific Research and by the EC-IHP project "Breaking Complexity". 
consider bases $\Psi$ of sufficiently smooth wavelet type of order $d$. Then the theory of nonlinear approximation ( $\mathrm{DeV98}$, Coh03]) tells us that if both

$$
0<s<\frac{d-t}{n}
$$

and $u$ is in the Besov space $B_{\tau}^{s n+t}\left(L_{\tau}\right)$, then

$$
\sup _{N \in \mathbb{N}} N^{s}\left\|\mathbf{u}-\mathbf{u}_{N}\right\|<\infty .
$$

Here, and throughout in this paper, $s$ and $\tau$ are related according to

$$
\tau=\left(\frac{1}{2}+s\right)^{-1} \text {. }
$$

The above condition involving Besov regularity is much milder than the condition $u \in H^{s n+t}$ involving Sobolev regularity which would be needed to guarantee the same rate of convergence with linear approximation in the span of $N$ wavelets corresponding to the "coarsest levels". Indeed, assuming a sufficiently smooth righthand side, for several boundary value problems it was proven that the solution has a much higher Besov than Sobolev regularity [DD97, Dah99. Note that, regardless of the smoothness of the solution $u$, a rate higher than $\frac{d-t}{n}$ can never be expected with wavelets of order $d$, except when $u$ happens to be exceptionally close to a finite linear combination of wavelets. On general domains or manifolds, suitable wavelet bases for $H^{t}$ have been constructed in [DS99a, CTU99, CM00, DS99b, Ste06, HS06].

Vectors $\mathbf{u} \in \ell_{2}$ that satisfy (1.1) can be characterized as follows (see DeV98): Let $\gamma_{n}(\mathbf{u})$ denote the $n$th largest coefficient in modulus of $\mathbf{u}$. For $0<\tau<2$, the space $\ell_{\tau}^{w}=\ell_{\tau}^{w}(\nabla)$ is defined by

$$
\ell_{\tau}^{w}=\left\{\mathbf{u} \in \ell_{2}:|\mathbf{u}|_{\ell_{\tau}^{w}}:=\sup _{n} n^{1 / \tau}\left|\gamma_{n}(\mathbf{u})\right|<\infty\right\} .
$$

It is easily verified that $\ell_{\tau} \hookrightarrow \ell_{\tau}^{w} \hookrightarrow \ell_{\tau+\delta}$ for any $\delta \in(0,2-\tau]$, which justifies why $\ell_{\tau}^{w}$ is called weak $\ell_{\tau}$. The expression $|\mathbf{u}|_{\ell_{\tau}^{w}}$ defines only a quasi-norm since it does not necessarily satisfy the triangle inequality. With these $\ell_{\tau}^{w}$-spaces at hand, it can be shown that the property (1.1) is equivalent to $\mathbf{u} \in \ell_{\tau}^{w}$. In particular, for each $\tau \in(0,2)$,

$$
\sup _{N} N^{s}\left\|\mathbf{u}-\mathbf{u}_{N}\right\| \bar{\sim}|\mathbf{u}|_{\ell_{\tau}^{w}}
$$

see, e.g., CDD01, Proposition 3.2]. Here and in the following, in order to avoid the repeated use of generic but unspecified constants, by $C \lesssim D$ we mean that $C$ can be bounded by a multiple of $D$, independently of parameters which $C$ and $D$ may depend on. Obviously, $C \gtrsim D$ is defined as $D \lesssim C$, and $C \approx D$ as $C \lesssim D$ and $C \gtrsim D$.

The aforementioned convergence rates under the mild Besov regularity assumption concern best $N$-term approximations, whose computation, however, requires full knowledge of the solution $\mathbf{u}$, which is only implicitly given. In CDD01, CDD02, iterative methods for solving $\mathbf{A u}=\mathbf{f}$ were developed which produce a sequence of approximations that converges with the same rate as is guaranteed for best $N$-term approximations, whereas their computation requires a number of operations that is equivalent to their support size. Together, both properties show that these methods are of optimal computational complexity. As a preparation for the results that will be derived in this paper, below we discuss both methods in some detail. 
In each iteration of these methods, the matrix $\mathbf{A}$ has to be applied to some (finitely supported) vector. Since, generally, each column of A contains infinitely many nonzero entries, clearly this matrix-vector product cannot be computed exactly, and has to be approximated. For sufficiently smooth wavelets that have sufficiently many vanishing moments and for both differential operators with piecewise sufficiently smooth coefficients, or singular integral operators on sufficiently smooth manifolds, the results from [Ste04, GS06a, GS06b] show that for some $s^{*}>\frac{d-t}{n}, \mathbf{A}$ is $s^{*}$-computable. This means that for any $s<s^{*}$, for all $N \in \mathbb{N}$, there is an infinite matrix $\mathbf{A}_{N}$, having in each column $\mathcal{O}(N)$ nonzero entries, whose computations require $\mathcal{O}(N)$ operations, such that

$$
\left\|\mathbf{A}-\mathbf{A}_{N}\right\| \lesssim N^{-s}
$$

Using this result, the adaptive approximate matrix-vector product APPLY from CDD02 can be shown to have the following properties.

$\operatorname{APPLY}[\mathbf{w}, \varepsilon] \rightarrow \mathbf{z}$. Let $\varepsilon>0$ and $\mathbf{w}$ be finitely supported, then the output satisfies $\|\mathbf{A w}-\mathbf{z}\| \leq \varepsilon$. Moreover, for any $s<s^{*}$, \# $\operatorname{supp} \mathbf{z} \lesssim \varepsilon^{-1 / s}|\mathbf{w}|_{\ell_{\tau}^{w}}^{1 / s}$, where the number of arithmetic operations and storage locations used by this call is bounded by some absolute multiple of $\varepsilon^{-1 / s}|\mathbf{w}|_{\ell_{\tau}^{w}}^{1 / s}+\# \operatorname{supp} \mathbf{w}+1$.

Remark 1.1. In DHS05] a somewhat weaker condition than (1.3) is verified, that, however, is also sufficient to guarantee above properties of APPLY.

The construction of a sequence of approximations for $\mathbf{u}$ that converge with a certain rate requires the availability of a sequence of approximations for $\mathbf{f}$ that converge with at least that rate. It can be shown that for any $s<s^{*}$, if $\mathbf{u} \in \ell_{\tau}^{w}$, then $\mathbf{f} \in \ell_{\tau}^{w}$, with $|\mathbf{f}|_{\ell_{\tau}^{w}} \lesssim|\mathbf{u}|_{\ell_{\tau}^{w}}$, and so $\sup _{N} N^{s}\left\|\mathbf{f}-\mathbf{f}_{N}\right\| \lesssim|\mathbf{u}|_{\ell_{\tau}^{w}}$. This property, however, does not tell us how to construct an approximation $\mathbf{g}$ that is qualitatively as good as $\mathbf{f}_{N}$ with a comparable support size. When $\mathbf{u} \in \ell_{\tau}^{w}$, we will assume the availability of the following routine, whose realization depends on the right-hand side at hand.

$\mathbf{R H S}[\varepsilon] \rightarrow \mathbf{g}$. Let $\varepsilon>0$, then the output satisfies $\|\mathbf{f}-\mathbf{g}\| \leq \varepsilon$, and \# $\operatorname{supp} \mathbf{g} \lesssim$ $\varepsilon^{-1 / s}|\mathbf{u}|_{\ell_{\tau}^{w}}^{1 / s}$, where moreover the number of arithmetic operations and storage locations used by the call is bounded by some absolute multiple of $\varepsilon^{-1 / s}|\mathbf{u}|_{\ell_{\tau}^{w}}^{1 / s}+1$.

The results concerning optimal computational complexity of the iterative methods from CDD01, CDD02 require the properties of APPLY and RHS mentioned above. Moreover, the methods apply under the condition that $\mathbf{A}$ is symmetric, positive definite (SPD), which, since $\mathbf{A}=\langle\Psi, A \Psi\rangle$, is equivalent to $\langle v, A w\rangle=\langle A v, w\rangle$, $v, w \in H$, and $\langle v, A v\rangle \gtrsim\|v\|_{H}^{2}, v \in H$.

For the case that $A$ does not have both properties, the methods can be applied to the normal equations $\mathbf{A}^{*} \mathbf{A u}=\mathbf{A}^{*} \mathbf{f}$. Using APPLY and RHS, in CDD02, Sect. 7] it was shown how to construct routines to approximate the matrix-vector product and the right-hand side vector of this system, which routines share the properties APPLY and RHS have for the original system. In other words, without loss of generality, in the following it is sufficient to consider the case that $\mathbf{A}$ is SPD.

The idea of the iterative method from CDD02 is to apply Richardson iteration to $\mathbf{A u}=\mathbf{f}$. Of course, this iteration cannot be performed exactly, but by ensuring that the errors due to the inexact matrix-vector product and the approximation of f exhibit a proper decay when the iteration proceeds, a linearly convergent method is obtained. 
The principle behind the method from CDD01 is to improve a given approximation $\mathbf{w}$ for $\mathbf{u}$ by realizing the saturation property: Let $\langle\langle\cdot, \cdot\rangle\rangle:=\langle\mathbf{A} \cdot, \cdot\rangle$ and $\|\cdot\|:=\langle\langle\cdot, \cdot\rangle\rangle^{\frac{1}{2}}$. For any $\Lambda \subset \nabla$, let $\mathbf{P}_{\Lambda}$ denote the $\ell_{2}$-orthogonal projector onto $\ell_{2}(\Lambda)$, i.e., $\mathbf{P}_{\Lambda}$ replaces all coefficients outside $\Lambda$ by zeros. With the notation $\mathbf{v}_{\Lambda}$, $\mathbf{z}_{\Lambda}$, etc., we will mean vectors in $\ell_{2}(\Lambda)$, i.e., vectors that are zero outside $\Lambda$. Using that $\mathbf{A}$ is SPD, one easily verifies that for any $\mathbf{v} \in \ell_{2}, \Lambda \subset \nabla$, and $\mathbf{v}_{\Lambda} \in \ell_{2}(\Lambda)$,

$$
\begin{gathered}
\left\|\mathbf{A}^{-1}\right\|^{-\frac{1}{2}}\|\mathbf{v}\| \leq\|\mathbf{v}\| \leq\|\mathbf{A}\|^{\frac{1}{2}}\|\mathbf{v}\|, \\
\|\mathbf{A} \mathbf{v}\| \leq\|\mathbf{A}\|^{\frac{1}{2}}\|\mathbf{v}\| \\
\left\|\mathbf{A}^{-1}\right\|^{-\frac{1}{2}}\left\|\mathbf{v}_{\Lambda}\right\| \leq\left\|\mathbf{P}_{\Lambda} \mathbf{A} \mathbf{v}_{\Lambda}\right\|,
\end{gathered}
$$

which properties will be often used in the following. The next lemma is well known.

Lemma 1.2. Let $\mu \in(0,1], \mathbf{w} \in \ell_{2}, \nabla \supset \Lambda \supset \operatorname{supp} \mathbf{w}$ such that

$$
\left\|\mathbf{P}_{\Lambda}(\mathbf{f}-\mathbf{A w})\right\| \geq \mu\|\mathbf{f}-\mathbf{A w}\| \text {. }
$$

Then, for $\mathbf{u}_{\Lambda} \in \ell_{2}(\Lambda)$ being the solution of the Galerkin system $\mathbf{P}_{\Lambda} \mathbf{A} \mathbf{u}_{\Lambda}=\mathbf{P}_{\Lambda} \mathbf{f}$, and with $\kappa(\mathbf{A}):=\|\mathbf{A}\|\left\|\mathbf{A}^{-1}\right\|$, we have

$$
\left\|\mathbf{u}-\mathbf{u}_{\Lambda}\right\| \leq\left[1-\kappa(\mathbf{A})^{-1} \mu^{2}\right]^{\frac{1}{2}}\|\mathbf{u}-\mathbf{w}\|
$$

Proof. We have

$$
\begin{aligned}
\left\|\mathbf{u}_{\Lambda}-\mathbf{w}\right\| & \geq\|\mathbf{A}\|^{-\frac{1}{2}}\left\|\mathbf{A}\left(\mathbf{u}_{\Lambda}-\mathbf{w}\right)\right\| \geq\|\mathbf{A}\|^{-\frac{1}{2}}\left\|\mathbf{P}_{\Lambda}(\mathbf{f}-\mathbf{A} \mathbf{w})\right\| \\
& \geq\|\mathbf{A}\|^{-\frac{1}{2}} \mu\|\mathbf{f}-\mathbf{A} \mathbf{w}\| \geq \kappa(\mathbf{A})^{-\frac{1}{2}} \mu\|\mathbf{u}-\mathbf{w}\|,
\end{aligned}
$$

that, with $\kappa(\mathbf{A})^{-\frac{1}{2}} \mu$ reading as some arbitrary positive constant, is known as the saturation property of the space $\ell_{2}(\Lambda)$ containing $\mathbf{w}$. The proof is completed by using the Galerkin orthogonality $\|\mathbf{u}-\mathbf{w}\|^{2}=\left\|\mathbf{u}-\mathbf{u}_{\Lambda}\right\|^{2}+\left\|\mathbf{u}_{\Lambda}-\mathbf{w}\right\|^{2}$.

In this lemma we assumed full knowledge about the exact residual, and furthermore that the arising Galerkin system is solved exactly. As with the Richardson iteration, however, linear convergence is retained with an inexact evaluation of the residuals and an inexact solution of the Galerkin systems in case the tolerances exhibit a proper decay as the iteration proceeds.

We remark that if, instead of being a Riesz basis, $\Psi$ is only a frame for $H$, then the inexact Richardson method is still applicable (see [Ste03, DFR04]); whereas, the other method is not since in that case the Galerkin systems can be arbitrarily badly conditioned.

Returning to the Riesz basis case, both iterative methods above are linearly convergent; however, their rates generally are not as good as that of best $N$-term approximations. Therefore, in CDD01, CDD02 these methods were extended with a so-called coarsening routine. After each $K$ iterations, where $K$ is a sufficiently large fixed constant, the smallest coefficients from the current iterand are removed, increasing the upper bound for its error with some factor larger than 2, but with that restoring the optimal balance between accuracy and vector length. Only after the extension with the coarsening routine, could the resulting methods be shown to be of optimal computational complexity.

In this paper, we reconsider the method from [CDD01]. Since for any subset $\Lambda \subset \nabla$ the best approximation in energy norm from $\ell_{2}(\Lambda)$ is the Galerkin solution that can be accurately approximated at relatively low cost, we expect that this 
method gives the best results quantitatively. The main point of the paper is that we will show that if $\mu$ is less than $\kappa(\mathbf{A})^{-\frac{1}{2}}$ and $\Lambda$ is the smallest set containing supp w that satisfies (1.4), then, without coarsening of the iterands, these approximations converge with a rate that is guaranteed for the best $N$-term approximations. Both conditions on the selection of $\Lambda$ can be qualitatively understood as follows. The basis of Lemma 1.2 is the use of the coefficients of the residual vector as local error indicators. In case $\kappa(\mathbf{A})=1$, the residual is just a multiple of the error. But when $\kappa(\mathbf{A}) \gg 1$, only the really largest coefficients can be used as reliable indicators about where the error is large. Of course, applying a larger set of indicators cannot reduce the convergence rate, but it may hamper optimal computational complexity. Notice the similarity with adaptive finite element methods where the largest local error indicators are used for marking elements for further refinement.

As we will see, the result above holds also true when the residuals and the Galerkin solutions are determined only inexactly, assuming a proper decay of the tolerances as the iteration proceeds, and when the cardinality of $\Lambda \backslash$ supp $\mathbf{w}$ is only minimal modulo some constant factor. Using both generalizations, again a method of optimal computational complexity is obtained.

One might argue that picking the largest coefficients of the (approximate) residual vector is another instance of coarsening, but on a different place in the algorithm. The principle behind it, however, is very different from that behind coarsening of the iterands. Furthermore, since with the new method no information is deleted that has been created by a sequence of computations, we expect that it is more efficient.

Another modification to the method from [CDD01] we will make is that for each call of APPLY or RHS, we will use as a tolerance some fixed multiple of the norm of the current approximate residual, instead of using an a priori prescribed tolerance. Since it seems hard to avoid that a priori tolerances are increasingly either unnecessarily small, making the calls costly, or large so that the perturbed iteration due to the inexact evaluations converges significantly slower than the unperturbed one, also here we expect to obtain a quantitative improvement.

We tested our adaptive wavelet solver for the Poisson equation on the interval. The results reported in the last section show that in this simple example the new method is indeed much more efficient than the inexact Richardson method with coarsening of the iterands. In DHS05, co-authored by the second author, numerical results based on tree approximations are given for singular integral equations on the boundary of three dimensional domains.

\section{The ADAPtive Method Without COARSENING OF THE ITERANDS}

In the following lemma it is shown that for sufficiently small $\mu$ and $\mathbf{u} \in \ell_{\tau}^{w}$, for a set $\Lambda$ as in Lemma 1.2 that has minimal cardinality, \#( $\Lambda \backslash \operatorname{supp} \mathbf{w})$ can be bounded in terms of $\|\mathbf{f}-\mathbf{A w}\|$ and $|\mathbf{u}|_{\ell_{\tau}^{w}}$ only, i.e., independently of $|\mathbf{w}|_{\ell_{\tau}^{w}}$ and the value of $s^{*}$ (cf. CDD01, §4.2-4.3]).

Lemma 2.1. Let $\mu \in\left(0, \kappa(\mathbf{A})^{-\frac{1}{2}}\right)$ be a constant, $\mathbf{w} \in \ell_{2}$, and for some $s>0$, $\mathbf{u} \in \ell_{\tau}^{w}$. Then the smallest set $\Lambda \supset \operatorname{supp} \mathbf{w}$ with

$$
\left\|\mathbf{P}_{\Lambda}(\mathbf{f}-\mathbf{A w})\right\| \geq \mu\|\mathbf{f}-\mathbf{A w}\|
$$


satisfies

$$
\#(\Lambda \backslash \operatorname{supp} \mathbf{w}) \lesssim\|\mathbf{f}-\mathbf{A w}\|^{-1 / s}|\mathbf{u}|_{\ell_{\tau}^{w}}^{1 / s} .
$$

Proof. Let $\lambda>0$ be a constant with $\mu \leq \kappa(\mathbf{A})^{-\frac{1}{2}}\left(1-\|\mathbf{A}\| \lambda^{2}\right)^{\frac{1}{2}}$. Let $N$ be the smallest integer such that a best $N$-term approximation $\mathbf{u}_{N}$ for $\mathbf{u}$ satisfies $\left\|\mathbf{u}-\mathbf{u}_{N}\right\| \leq \lambda\|\mathbf{u}-\mathbf{w}\|$. Since $\|\mathbf{u}-\mathbf{w}\| \geq\|\mathbf{A}\|^{-\frac{1}{2}}\|\mathbf{f}-\mathbf{A} \mathbf{w}\|$, we have

$$
N \lesssim\|\mathbf{f}-\mathbf{A w}\|^{-1 / s}|\mathbf{u}|_{\ell_{\tau}^{w}}^{1 / s}
$$

With $\breve{\Lambda}:=\operatorname{supp} \mathbf{w} \cup \operatorname{supp} \mathbf{u}_{N}$, the solution of $\mathbf{P}_{\breve{\Lambda}} \mathbf{A} \mathbf{u}_{\breve{\Lambda}}=\mathbf{P}_{\breve{\Lambda}} \mathbf{f}$ satisfies

$$
\left\|\mathbf{u}-\mathbf{u}_{\Lambda}\right\| \leq\left\|\mathbf{u}-\mathbf{u}_{N}\right\| \leq\|\mathbf{A}\|^{\frac{1}{2}}\left\|\mathbf{u}-\mathbf{u}_{N}\right\| \leq\|\mathbf{A}\|^{\frac{1}{2}} \lambda\|\mathbf{u}-\mathbf{w}\|,
$$

and so by Galerkin orthogonality, $\left\|\mathbf{u}_{\breve{\Lambda}}-\mathbf{w}\right\| \geq\left(1-\|\mathbf{A}\| \lambda^{2}\right)^{\frac{1}{2}}\|\mathbf{u}-\mathbf{w}\|$, giving

$$
\begin{aligned}
\left\|\mathbf{P}_{\breve{\Lambda}}(\mathbf{f}-\mathbf{A} \mathbf{w})\right\| & =\left\|\mathbf{P}_{\breve{\Lambda}}\left(\mathbf{A} \mathbf{u}_{\breve{\Lambda}}-\mathbf{A} \mathbf{w}\right)\right\| \geq\left\|\mathbf{A}^{-1}\right\|^{-\frac{1}{2}}\left\|\mathbf{u}_{\breve{\Lambda}}-\mathbf{w}\right\| \\
& \geq\left\|\mathbf{A}^{-1}\right\|^{-\frac{1}{2}}\left(1-\|\mathbf{A}\| \lambda^{2}\right)^{\frac{1}{2}}\|\mathbf{u}-\mathbf{w}\| \\
& \geq \kappa(\mathbf{A})^{-\frac{1}{2}}\left(1-\|\mathbf{A}\| \lambda^{2}\right)^{\frac{1}{2}}\|\mathbf{f}-\mathbf{A} \mathbf{w}\| \\
& \geq \mu\|\mathbf{f}-\mathbf{A} \mathbf{w}\| .
\end{aligned}
$$

Since $\breve{\Lambda} \supset$ supp w, by definition of $\Lambda$ we conclude that

$$
\#(\Lambda \backslash \operatorname{supp} \mathbf{w}) \leq \#(\breve{\Lambda} \backslash \operatorname{supp} \mathbf{w}) \leq N \lesssim\|\mathbf{f}-\mathbf{A w}\|^{-1 / s}|\mathbf{u}|_{\ell_{\tau}^{w}}^{1 / s}
$$

Since we do not have access to the exact residual, obviously Lemma 2.1 cannot be applied directly. The following routine GROW provides a practical algorithm for extending the support of an approximation $\mathbf{w}$ for $\mathbf{u}$ to a set $\Lambda$, which is sufficiently large such that $\ell_{2}(\Lambda)$ has the saturation property, but whose cardinality can be bounded as in Lemma 2.1. Firstly, inside a loop, the tolerances for the approximate matrix-vector product and the approximation of the right-hand side are decreased until either the computed approximate residual $\mathbf{r}$ has a sufficiently small relative error, or the norm of the residual is below the target tolerance in which case $\mathbf{w}$ will be accepted as a valid approximation for $\mathbf{u}$. In case the norm of the residual is not below the target tolerance, secondly, a set $\Lambda \supset$ supp w is determined with modulo some constant factor minimal $\#(\Lambda \backslash \operatorname{supp} \mathbf{w})$ such that $\left\|\mathbf{P}_{\Lambda} \mathbf{r}\right\| \geq \alpha\|\mathbf{r}\|$.

GROW $[\mathbf{w}, \bar{\nu}, \varepsilon] \rightarrow[\Lambda, \nu]:$

$\%$ Let $\alpha, \omega$ be constants with $0<\omega<\alpha, \frac{\alpha+\omega}{1-\omega}<\kappa(\mathbf{A})^{-\frac{1}{2}}$.

$\zeta:=2 \frac{\omega \bar{\nu}}{1-\omega}$

do $\zeta:=\zeta / 2, \mathbf{r}:=\mathbf{R H S}[\zeta / 2]-\mathbf{A P P L Y}[\mathbf{w}, \zeta / 2]$

until $\nu:=\|\mathbf{r}\|+\zeta \leq \varepsilon$ or $\zeta \leq \omega\|\mathbf{r}\|$

if $\nu>\varepsilon$

then determine a set $\Lambda \supset$ supp $\mathbf{w}$ with modulo some absolute constant factor minimal $\#(\Lambda \backslash \operatorname{supp} \mathbf{w})$ such that $\left\|\mathbf{P}_{\Lambda} \mathbf{r}\right\| \geq \alpha\|\mathbf{r}\|$

else $\Lambda:=\emptyset$

endif

Remark 2.2. GROW will be called with a parameter $\bar{\nu}$ that estimates the norm of the residual of $\mathbf{w}$. If it is outside $\left[\frac{1-\omega}{1+\omega}\|\mathbf{f}-\mathbf{A w}\|,\|\mathbf{f}-\mathbf{A w}\|\right]$, then $\zeta$ at the first evaluation of $\mathbf{r}$ is outside $\left[\frac{\omega}{1+\omega}\|\mathbf{f}-\mathbf{A w}\|, \frac{\omega}{1-\omega}\|\mathbf{f}-\mathbf{A w}\|\right]$, and from $\omega\|\mathbf{f}-\mathbf{A w}\|-\zeta \leq$ $\omega\|\mathbf{r}\| \leq \omega\|\mathbf{f}+\mathbf{A w}\|+\zeta$, one infers that in this case either the second test in the until-clause will fail anyway, meaning that the first iteration of the do-loop is not of 
any use, or that second test in the until-clause is always passed, but possibly with a tolerance that is unnecessarily small. We conclude that there is not much sense in calling GROW with a value of $\bar{\nu}$ that is far outside $\left[\frac{1-\omega}{1+\omega}\|\mathbf{f}-\mathbf{A} \mathbf{w}\|,\|\mathbf{f}-\mathbf{A} \mathbf{w}\|\right]$.

Remark 2.3. Selecting $\Lambda$ in GROW with truly minimal cardinality would require the sorting of all coefficients of $\left.\mathbf{r}\right|_{\nabla \backslash \text { supp w }}$ by their modulus, which needs $\mathcal{O}(N \log N)$ operations with $N:=\left.\# \operatorname{supp} \mathbf{r}\right|_{\nabla \backslash \operatorname{supp} \mathbf{w}}$. Another $\mathcal{O}(\# \operatorname{supp} \mathbf{r})$ operations for computing $\|\mathbf{r}\|$ are unavoidable. In the following, we recall a procedure with which the above log-factor is avoided.

In view of our task to select $\Lambda \supset \operatorname{supp} \mathbf{w}$ with $\left\|\mathbf{P}_{\Lambda} \mathbf{r}\right\| \geq \alpha\|\mathbf{r}\|$, we may discard all coefficients of $\left.\mathbf{r}\right|_{\nabla \backslash \text { supp w }}$ with modulus not larger than $\sqrt{\left(1-\alpha^{2}\right)}\|\mathbf{r}\| / \sqrt{N}$. With $M:=\left\|\left.\mathbf{r}\right|_{\nabla \backslash \text { supp } \mathbf{w}}\right\|_{\infty}$, and $q$ being the smallest integer with $2^{-(q+1) / 2} M \leq$ $\sqrt{\left(1-\alpha^{2}\right)}\|\mathbf{r}\| / \sqrt{N}$, we store the other coefficients of $\left.\mathbf{r}\right|_{\nabla \backslash \text { supp } \mathbf{w}}$ in $q+1$ bins corresponding whether they lie in $\left[M, \frac{1}{\sqrt{2}} M\right),\left[\frac{1}{\sqrt{2}} M, \frac{1}{2} M\right), \ldots$, or $\left[2^{-q / 2} M, 2^{-(q+1) / 2} M\right)$. Then we build $\Lambda$ by extracting coefficients from the bins, starting with the first bin, and when it is empty moving to the second bin and so on until $\left\|\mathbf{P}_{\Lambda} \mathbf{r}\right\| \geq \alpha\|\mathbf{r}\|$ is satisfied. Let the resulting $\Lambda$ now contain coefficients from the $p$ th bin, but not from further bins. Then a minimal set $\tilde{\Lambda}$ that satisfies $\left\|\mathbf{P}_{\tilde{\Lambda}} \mathbf{r}\right\| \geq \alpha\|\mathbf{r}\|$ contains all coefficients from the bins up to the $(p-1)$ th one. Since any two coefficients in the $p$ th bin differ less than a factor $\sqrt{2}$, we infer that the cardinality of the contribution from the $p$ th bin to $\Lambda$ is at most twice as large as that to $\tilde{\Lambda}$, so that $\#(\Lambda \backslash \operatorname{supp} \mathbf{w}) \leq 2 \#(\tilde{\Lambda} \backslash \operatorname{supp} \mathbf{w})$. The number of operations and storage locations required by this procedure is $\mathcal{O}(\# \operatorname{supp} \mathbf{r}+q)$, where $q<2 \log _{2}\left(M \sqrt{N} /\left[\sqrt{1-\alpha^{2}}\|\mathbf{r}\|\right]\right) \leq 2 \log _{2}\left(\sqrt{N} / \sqrt{1-\alpha^{2}}\right) \lesssim \log _{2}(\sqrt{N})<\#$ supp r.

In the next theorem it is shown that if $\mathbf{G R O W}[\mathbf{w}, \bar{\nu}, \varepsilon]$ does not terminate because the target tolerance $\varepsilon$ is met, then it outputs a set $\Lambda$ that satisfies both (1.4) with $\mu=\frac{\alpha-\omega}{1+\omega}$ and (2.1).

Theorem 2.4. $[\Lambda, \nu]=\mathbf{G R O W}[\mathbf{w}, \bar{\nu}, \varepsilon]$ terminates with $\nu \geq\|\mathbf{f}-\mathbf{A w}\|$ and $\nu \gtrsim$ $\min \{\bar{\nu}, \varepsilon\}$. If, for some $s<s^{*}, \mathbf{u} \in \ell_{\tau}^{w}$, then the number of arithmetic operations and storage locations required by the call is bounded by some absolute multiple of $\min \{\bar{\nu}, \nu\}^{-1 / s}\left[|\mathbf{w}|_{\ell_{\tau}^{w}}^{1 / s}+|\mathbf{u}|_{\ell_{\tau}^{w}}^{1 / s}+\bar{\nu}^{1 / s}(\# \operatorname{supp} \mathbf{w}+1)\right]$.

If GROW terminates with $\nu>\varepsilon$, then

$$
\frac{\alpha-\omega}{1+\omega} \nu \leq\left\|\mathbf{P}_{\Lambda}(\mathbf{f}-\mathbf{A w})\right\|
$$

and

$$
\#(\Lambda \backslash \operatorname{supp} \mathbf{w}) \lesssim \nu^{-1 / s}|\mathbf{u}|_{\ell_{\tau}^{w}}^{1 / s} .
$$

Proof. If, at evaluation of the until-clause $\zeta>\omega\|\mathbf{r}\|$, then $\|\mathbf{r}\|+\zeta<\left(\omega^{-1}+1\right) \zeta$. Since $\zeta$ is halved in each iteration, we infer that, if not by $\zeta \leq \omega\|\mathbf{r}\|$, GROW will terminate by $\|\mathbf{r}\|+\zeta \leq \varepsilon$.

Since after any evaluation of $\mathbf{r}$ inside the algorithm, $\|\mathbf{r}-(\mathbf{f}-\mathbf{A w})\| \leq \zeta$, any value of $\nu$ determined inside the algorithm is an upper bound on $\|\mathbf{f}-\mathbf{A w}\|$. If the do-loop terminates in the first iteration or the algorithm terminates with $\nu>\varepsilon$, then $\nu \gtrsim \min \{\bar{\nu}, \varepsilon\}$. In the other case, let $\mathbf{r}^{\text {old }}:=\mathbf{R H S}[\zeta]-\mathbf{A P P L Y}[\mathbf{w}, \zeta]$. We have $\left\|\mathbf{r}^{\text {old }}\right\|+2 \zeta>\varepsilon$ and $2 \zeta>\omega\left\|\mathbf{r}^{\text {old }}\right\|$, so that $\nu \geq \zeta>\left(2 \omega^{-1}+2\right)^{-1}\left(\left\|\mathbf{r}^{\text {old }}\right\|+2 \zeta\right)>\frac{\omega \varepsilon}{2+2 \omega}$.

By the geometrical decrease of $\zeta$ inside the algorithm, the properties of RHS and APPLY, and in view Remark 2.3, the total cost of the call of GROW can 
be bounded by some multiple of $\zeta^{-1 / s}\left(|\mathbf{w}|_{\ell_{\tau}^{w}}^{1 / s}+|\mathbf{u}|_{\ell_{\tau}^{w}}^{1 / s}\right)+K(\# \operatorname{supp} \mathbf{w}+1)$, with $\zeta, \mathbf{r}$ and $\nu$ having their values at termination and $K^{\tau}$ being the number of calls of APPLY that were made. Taking into account the initial value of $\zeta$, and again its geometrical decrease inside the algorithm, we have

$$
K(\# \operatorname{supp} \mathbf{w}+1)=K \bar{\nu}^{-1 / s} \bar{\nu}^{1 / s}(\# \operatorname{supp} \mathbf{w}+1) \lesssim \zeta^{-1 / s} \bar{\nu}^{1 / s}(\# \operatorname{supp} \mathbf{w}+1) .
$$

The proof of the first part of the theorem is completed once we have shown that $\zeta \gtrsim \min \{\bar{\nu}, \nu\}$. When the do-loop terminates in the first iteration, we have $\zeta \gtrsim \bar{\nu}$, and when the algorithm terminates with $\zeta \geq \omega\|\mathbf{r}\|$, we have $\zeta \gtrsim \nu$. In the other case, we have $\omega\left\|\mathbf{r}^{\text {old }}\right\|<2 \zeta$ with $\mathbf{r}^{\text {old }}$ as above, and so from $\left\|\mathbf{r}-\widetilde{\mathbf{r}}^{\text {old }}\right\| \leq \zeta+2 \zeta$, we infer $\|\mathbf{r}\| \leq\left\|\mathbf{r}^{\text {old }}\right\|+3 \zeta<\left(2 \omega^{-1}+3\right) \zeta$, so that $\nu<\left(2 \omega^{-1}+4\right) \zeta$.

Now assume that GROW terminates with $\nu>\varepsilon$ and thus with $\zeta \leq \omega\|\mathbf{r}\|$. With $\mathbf{g}=\mathbf{R H S}[\zeta / 2]$ and $\mathbf{z}=\mathbf{A P P L Y}[\mathbf{w}, \zeta / 2]$, we have

$$
\begin{aligned}
\left\|\mathbf{P}_{\Lambda}(\mathbf{f}-\mathbf{A} \mathbf{w})\right\| & \geq\left\|\mathbf{P}_{\Lambda} \mathbf{r}\right\|-\left\|\mathbf{P}_{\Lambda}(\mathbf{A} \mathbf{w}-\mathbf{z})\right\|-\left\|\mathbf{P}_{\Lambda}(\mathbf{f}-\mathbf{g})\right\| \\
& \geq \alpha\|\mathbf{r}\|-\zeta \geq \frac{\alpha-\omega}{1+\omega} \nu,
\end{aligned}
$$

where the last inequality is a consequence of $\zeta \leq \omega\|\mathbf{r}\|, \omega<\alpha$, and $\nu=\|\mathbf{r}\|+\zeta$.

To prove (2.3), with $\mu=\frac{\alpha+\omega}{1-\omega}$ let $\hat{\Lambda} \supset$ supp w be the smallest set with

$$
\left\|\mathbf{P}_{\hat{\Lambda}}(\mathbf{f}-\mathbf{A w})\right\| \geq \mu\|\mathbf{f}-\mathbf{A w}\| \text {. }
$$

Then

$$
\mu\|\mathbf{r}\| \leq \mu\|\mathbf{f}-\mathbf{A} \mathbf{w}\|+\mu \zeta \leq\left\|\mathbf{P}_{\hat{\Lambda}}(\mathbf{f}-\mathbf{A} \mathbf{w})\right\|+\mu \zeta \leq\left\|\mathbf{P}_{\hat{\Lambda}} \mathbf{r}\right\|+(1+\mu) \omega\|\mathbf{r}\|,
$$

or $\left\|\mathbf{P}_{\hat{\Lambda}} \mathbf{r}\right\| \geq \alpha\|\mathbf{r}\|$. By construction of $\Lambda$ in GROW, we conclude that $\#(\Lambda \backslash$ supp w $)$ $\lesssim \#(\hat{\Lambda} \backslash \operatorname{supp} \mathbf{w})$. Since $\mu<\kappa(\mathbf{A})^{-\frac{1}{2}}$ by the condition on $\omega$ and $\alpha$, and $\|\mathbf{f}-\mathbf{A} \mathbf{w}\|$ $\leq \nu$, an application of Lemma 2.1 shows that $\#(\hat{\Lambda} \backslash \operatorname{suppw}) \lesssim \nu^{-1 / s}|\mathbf{u}|_{\ell_{\tau}^{w}}^{1 / s}$ which completes the proof.

When having extended supp w to a set $\Lambda$ such that $\ell_{2}(\Lambda)$ has the saturation property, the second ingredient of the iterative method is the approximate solution of the Galerkin system on $\ell_{2}(\Lambda)$. Given an approximation $\mathbf{g}_{\Lambda}$ for $\mathbf{P}_{\Lambda} \mathbf{f}$, there are various possibilities to iteratively solving the system $\mathbf{P}_{\Lambda} \mathbf{A} \mathbf{u}_{\Lambda}=\mathbf{g}_{\Lambda}$ starting with some initial approximation $\mathbf{w}_{\Lambda}$ for $\mathbf{u}_{\Lambda}$, where obviously we will take $\mathbf{w}_{\Lambda}=\mathbf{w}$. Instead of relying on the adaptive routine APPLY throughout the iteration, after approximately computing the initial residual using the APPLY routine, the following routine GALSOLVE iterates using some fixed, nonadaptive approximation for

$$
\mathbf{A}_{\Lambda}:=\left.\mathbf{P}_{\Lambda} \mathbf{A}\right|_{\ell_{2}(\Lambda)}
$$

The accuracy of this approximation depends only on the factor with which one wants to reduce the norm of the residual. This approach can be expected to be particularly efficient when the approximate computation of the entries of $\mathbf{A}$ is relatively expensive, as with singular integral operators. As can be deduced from vS04, it is even possible in the course of the iteration to gradually diminish the accuracy of the approximation for $\mathbf{A}_{\Lambda}$.

$\operatorname{GALSOLVE}\left[\Lambda, \mathbf{g}_{\Lambda}, \mathbf{w}_{\Lambda}, \delta, \varepsilon\right] \rightarrow \tilde{\mathbf{w}}_{\Lambda}:$ $\%$ The input should satisfy $\delta \geq\left\|\mathbf{g}_{\Lambda}-\mathbf{A}_{\Lambda} \mathbf{w}_{\Lambda}\right\|$.

$\%$ With $\mathbf{A}_{N}$ from (1.3), let $N$ be such that $\sigma:=\left\|\mathbf{A}-\mathbf{A}_{N}\right\|\left\|\mathbf{A}^{-1}\right\| \leq \frac{\varepsilon}{3 \varepsilon+3 \delta}$.

$\%$ Set $\mathbf{B}:=\left.\mathbf{P}_{\Lambda} \frac{1}{2}\left(\mathbf{A}_{N}+\mathbf{A}_{N}^{*}\right)\right|_{\ell_{2}(\Lambda)}$, with $\mathbf{A}_{N}^{*}$ being the adjoint of $\mathbf{A}_{N}$.

$\mathbf{r}_{0}:=\mathbf{g}_{\Lambda}-\mathbf{P}_{\Lambda}\left(\mathbf{A P P L Y}\left[\mathbf{w}_{\Lambda}, \frac{\varepsilon}{3}\right]\right)$ 
To find an $\mathbf{x}$ with $\left\|\mathbf{r}_{0}-\mathbf{B} \mathbf{x}\right\| \leq \frac{\varepsilon}{3}$, apply a suitable iterative method for solving $\mathbf{B} \mathbf{x}=\mathbf{r}_{0}$, e.g., Conjugate Gradients or Conjugate Residuals $\tilde{\mathbf{w}}_{\Lambda}:=\mathbf{w}_{\Lambda}+\mathbf{x}$

Theorem 2.5. $\tilde{\mathbf{w}}_{\Lambda}:=\operatorname{GALSOLVE}\left[\Lambda, \mathbf{g}_{\Lambda}, \mathbf{w}_{\Lambda}, \delta, \varepsilon\right]$ satisfies $\left\|\mathbf{g}_{\Lambda}-\mathbf{A}_{\Lambda} \tilde{\mathbf{w}}_{\Lambda}\right\| \leq$ $\varepsilon$. For any $s<s^{*}$, the number of arithmetic operations and storage locations required by the call is bounded by some absolute multiple of $\varepsilon^{-1 / s}\left(\left|\mathbf{w}_{\Lambda}\right|_{\ell_{\tau}^{w}}^{1 / s}+\left|\mathbf{u}_{\Lambda}\right|_{\ell_{\tau}^{w}}^{1 / s}\right)+$ $c(\delta / \varepsilon) \# \Lambda$, where $c: \mathbb{R}_{+} \rightarrow \mathbb{R}_{+}$is some nondecreasing function.

Proof. Using $\left\langle\mathbf{A}_{\Lambda} \mathbf{v}_{\Lambda}, \mathbf{v}_{\Lambda}\right\rangle \geq\left\|\mathbf{A}^{-1}\right\|^{-1}\left\|\mathbf{v}_{\Lambda}\right\|^{2}$ and $\left\|\mathbf{A}_{\Lambda}-\mathbf{B}\right\| \leq\left\|\mathbf{A}-\mathbf{A}_{N}\right\|=\sigma\left\|\mathbf{A}^{-1}\right\|^{-1}$ $<\frac{1}{3}\left\|\mathbf{A}^{-1}\right\|^{-1}$, we infer that $\mathbf{B}$ is SPD with respect to the canonical scalar product on $\ell_{2}(\Lambda)$, and that $\kappa(\mathbf{B}) \lesssim 1$ uniformly in $\varepsilon$ and $\delta$. Writing $\mathbf{B}^{-1}=$ $\left(\mathbf{I}-\mathbf{A}_{\Lambda}^{-1}\left(\mathbf{A}_{\Lambda}-\mathbf{B}\right)\right)^{-1} \mathbf{A}_{\Lambda}^{-1}$, we find that $\left\|\mathbf{B}^{-1}\right\| \leq \frac{\left\|\mathbf{A}_{\Lambda}^{-1}\right\|}{1-\left\|\mathbf{A}_{\Lambda}^{-1}\right\|\left\|\mathbf{A}_{\Lambda}-\mathbf{B}\right\|}$ and so that $\left\|\mathbf{A}_{\Lambda}-\mathbf{B}\right\|\left\|\mathbf{B}^{-1}\right\| \leq \frac{\sigma}{1-\sigma}$.

We have $\left\|\mathbf{r}_{0}\right\| \leq \delta+\frac{\varepsilon}{3}$. Writing

$$
\mathbf{g}_{\Lambda}-\mathbf{A}_{\Lambda} \tilde{\mathbf{w}}_{\Lambda}=\left(\mathbf{g}_{\Lambda}-\mathbf{A}_{\Lambda} \mathbf{w}_{\Lambda}-\mathbf{r}_{0}\right)+\left(\mathbf{r}_{0}-\mathbf{B x}\right)+\left(\mathbf{B}-\mathbf{A}_{\Lambda}\right) \mathbf{B}^{-1}\left(\mathbf{r}_{0}+\mathbf{B} \mathbf{x}-\mathbf{r}_{0}\right),
$$

we find that

$$
\left\|\mathbf{A}_{\Lambda} \tilde{\mathbf{w}}_{\Lambda}-\mathbf{g}_{\Lambda}\right\| \leq \frac{\varepsilon}{3}+\frac{\varepsilon}{3}+\frac{\sigma}{1-\sigma}\left(\delta+\frac{\varepsilon}{3}+\frac{\varepsilon}{3}\right) \leq \varepsilon .
$$

The properties of APPLY and RHS show that the cost of the computation of $\mathbf{r}_{0}$ is bounded by some multiple of $\varepsilon^{-1 / s}\left(\left|\mathbf{w}_{\Lambda}\right|_{\ell_{\tau}^{w}}^{1 / s}+\left|\mathbf{u}_{\Lambda}\right|_{\ell_{\tau}^{w}}^{1 / s}\right)+\# \Lambda$. Since by (1.3), $\mathbf{B}$ is sparse and can be constructed in $\mathcal{O}(\# \Lambda)$ operations, and the required number of iterations of the iterative method is bounded, everything only dependent on an upper bound for $\delta / \varepsilon$, the proof is completed.

We now have the ingredients available to define our adaptive wavelet solver.

$\operatorname{SOLVE}\left[\nu_{-1}, \varepsilon\right] \rightarrow \mathbf{w}_{k}:$

$\%$ With $\alpha, \omega$ being the parameters inside GROW, let $\gamma$ be a constant in $\%\left(0, \frac{1}{6} \kappa(\mathbf{A})^{-\frac{1}{2}} \frac{\alpha-\omega}{1+\omega}\right)$.

$\%$ Let $\theta>0$ be a constant.

$k:=0 ; \mathbf{w}_{k}:=0$

while with $\left[\Lambda_{k+1}, \nu_{k}\right]:=\mathbf{G R O W}\left[\mathbf{w}_{k}, \theta \nu_{k-1}, \varepsilon\right], \nu_{k}>\varepsilon$ do

$\mathbf{g}_{k+1}:=\mathbf{P}_{\Lambda_{k+1}}\left(\mathbf{R H S}\left[\gamma \nu_{k}\right]\right)$

$\mathbf{w}_{k+1}:=\mathbf{G A L S O L V E}\left[\Lambda_{k+1}, \mathbf{g}_{k+1}, \mathbf{w}_{k},(1+\gamma) \nu_{k}, \gamma \nu_{k}\right]$

$k:=k+1$

enddo

Remark 2.6. We will see that at the call of $\mathbf{G R O W}\left[\mathbf{w}_{k}, \theta \nu_{k-1}, \varepsilon\right]$, it holds that $\left\|\mathbf{f}-\mathbf{A w}_{k}\right\| \lesssim \nu_{k-1}$. Although for any fixed $\theta>0$, SOLVE will be shown to be of optimal computational complexity, in view of Remark 2.2 a suitable tuning of $\theta$ will result in quantitatively better results. Ideally, $\theta$ has the largest value for which the do-loop inside GROW always terminates in one iteration.

Theorem 2.7. $\mathbf{w}:=\mathbf{S O L V E}\left[\nu_{-1}, \varepsilon\right]$ terminates with $\|\mathbf{A w}-\mathbf{f}\| \leq \varepsilon$. If $\nu_{-1} \bar{\sim}$ $\|\mathbf{f}\| \gtrsim \varepsilon$, and for some $s<s^{*}, \mathbf{u} \in \ell_{\tau}^{w}$, then \# $\operatorname{supp} \mathbf{w} \lesssim \varepsilon^{-1 / s}|\mathbf{u}|_{\ell_{\tau}^{w}}^{1 / s}$ and the number of arithmetic operations and storage locations required by the call is bounded by some absolute multiple of the same expression.

Proof. Before we come to the actual proof, first we indicate the need for the conditions involving $\nu_{-1},\|\mathbf{f}\|$ and $\varepsilon$. If $\nu_{-1} \gtrsim \varepsilon$, then the cost of the first call of RHS 
in the first call of GROW can be arbitrarily large. If $\nu_{-1} \not\|\mathbf{f}\|$, then we cannot bound the number of iterations in the loop of the first call of GROW, each of them requiring some arithmetic operations. Finally, if $\|\mathbf{f}\| \gtrsim \varepsilon$, then $\varepsilon^{-1 / s}|\mathbf{u}|_{\ell_{\tau}^{w}}^{1 / s}$ might be arbitrarily small, whereas SOLVE takes at least some arithmetic operations.

Theorem 2.4 shows that $\nu_{k} \geq\left\|\mathbf{A} \mathbf{w}_{k}-\mathbf{f}\right\|$, and that $\nu_{k} \lesssim\left\|\mathbf{f}-\mathbf{A} \mathbf{w}_{k}\right\|$ as long as $\nu_{k}>\varepsilon$. We have $\left\|\mathbf{g}_{k+1}-\mathbf{P}_{\Lambda_{k+1}} \mathbf{A} \mathbf{w}_{k}\right\| \leq(1+\gamma) \nu_{k}$, so that $(1+\gamma) \nu_{k}$ is a valid parameter for the $(k+1)$ th call of GALSOLVE. Below we will prove that a constant $\xi<1$ exists such that

$$
\left\|\mathbf{u}-\mathbf{w}_{k+1}\right\| \leq \xi\left\|\mathbf{u}-\mathbf{w}_{k}\right\|
$$

as long as $\nu_{k}>\varepsilon$. Because of $\left\|\mathbf{A} \mathbf{w}_{k}-\mathbf{f}\right\| \approx\left\|\mathbf{u}-\mathbf{w}_{k}\right\|$, this result shows that SOLVE terminates after finitely many iterations, say directly after the $(K+1)$ th call of GROW that produces $\left[\Lambda_{K+1}, \nu_{K}\right]$, and furthermore that $\nu_{k} \lesssim \xi^{k-i} \nu_{i}$ for all $0 \leq i \leq k \leq K-1$. From $\nu_{K} \leq \varepsilon<\nu_{K-1}$ when $K>0$, and $\nu_{0} \leq \max \left\{\varepsilon, \frac{1+\omega}{\alpha-\omega}\|\mathbf{f}\|\right\} \lesssim$ $\nu_{-1}$, the last inequality by assumption, we even have

$$
\nu_{k} \lesssim \xi^{k-i} \nu_{i}, \quad-1 \leq i \leq k \leq K
$$

Since with $\Lambda_{0}:=\emptyset$, supp $\mathbf{w}_{i} \subset \Lambda_{i}$ and $\Lambda_{i} \subset \Lambda_{i+1}$, for $1 \leq k \leq K$ by (2.3) we have

$$
\# \operatorname{supp} \mathbf{w}_{k} \leq \# \Lambda_{k}=\sum_{i=0}^{k-1} \#\left(\Lambda_{i+1} \backslash \Lambda_{i}\right) \lesssim\left(\sum_{i=0}^{k-1} \nu_{i}^{-1 / s}\right)|\mathbf{u}|_{\ell_{\tau}^{w}}^{1 / s} \lesssim \nu_{k-1}^{-1 / s}|\mathbf{u}|_{\ell_{\tau}^{w}}^{1 / s}
$$

From $\left|\mathbf{w}_{k}\right|_{\ell_{\tau}^{w}} \lesssim|\mathbf{u}|_{\ell_{\tau}^{w}}+\left(\# \operatorname{supp} \mathbf{w}_{k}\right)^{s}\left\|\mathbf{w}_{k}-\mathbf{u}\right\|$ (CDD01, Lemma 4.11]), we infer that $\left|\mathbf{w}_{k}\right|_{\ell_{\tau}^{w}} \lesssim|\mathbf{u}|_{\ell_{\tau}^{w}}$.

By Theorem 2.4 the cost of the $(k+1)$ th call of GROW for $k \leq K$ is bounded by an absolute multiple of

$$
\min \left\{\nu_{k-1}, \nu_{k}\right\}^{-1 / s}\left[|\mathbf{u}|_{\ell_{\tau}^{w}}^{1 / s}+\nu_{k-1}^{1 / s}\left(\nu_{k-1}^{-1 / s}|\mathbf{u}|_{\ell_{\tau}^{w}}^{1 / s}+1\right)\right] \lesssim \nu_{k}^{-1 / s}|\mathbf{u}|_{\ell_{\tau}^{w}}^{1 / s}
$$

where we used (2.8), $\min \left\{\nu_{k-1}, \nu_{k}\right\} \gtrsim \nu_{k}$ by (2.7), and $1 \lesssim \nu_{k-1}^{-1 / s}|\mathbf{u}|_{\ell_{\tau}^{w}}^{1 / s}$ by $\nu_{k-1} \lesssim$ $\nu_{-1} \lesssim\|\mathbf{f}\| \lesssim|\mathbf{u}|_{\ell_{\tau}^{w}}$. The cost of the $(k+1)$ th call for $k<K$ of RHS or GALSOLVE is bounded by an absolute multiple of $\nu_{k}^{-1 / s}|\mathbf{u}|_{\ell_{\tau}^{w}}^{1 / s}$ or $\nu_{k}^{-1 / s}\left(\left|\mathbf{w}_{k}\right|_{\ell_{\tau}^{w}}^{1 / s}+|\mathbf{u}|_{\ell_{\tau}^{w}}^{1 / s}\right)+$ $\# \Lambda_{k+1} \lesssim \nu_{k}^{-1 / s}|\mathbf{u}|_{\ell_{\tau}^{w}}^{1 / s}$, respectively. From (2.7) and $\nu_{K} \gtrsim \min \left\{\nu_{K-1}, \varepsilon\right\} \gtrsim \varepsilon$ by Theorem 2.4 where the second inequality follows from $\nu_{K-1}>\varepsilon$ when $K>0$, and by assumption when $K=0$, the proof is completed upon showing (2.6).

Abbreviating $\mathbf{P}_{\Lambda_{k+1}}$ as $\mathbf{P}_{k+1}$, for $0 \leq k<K$, let $\mathbf{u}_{k+1}$ be the solution of $\mathbf{P}_{k+1} \mathbf{A} \mathbf{u}_{k+1}=\mathbf{P}_{k+1} \mathbf{f}$. Because of $\left\|\mathbf{f}-\mathbf{A} \mathbf{w}_{k}\right\| \leq \nu_{k}$ and (2.2), that can be applied since $\nu_{k}>\varepsilon$, we have $\left\|\mathbf{P}_{k+1}\left(\mathbf{f}-\mathbf{A} \mathbf{w}_{k}\right)\right\| \geq \frac{\alpha-\omega}{1+\omega}\left\|\mathbf{f}-\mathbf{A} \mathbf{w}_{k}\right\|$, so that Lemma 1.2 shows that $\left\|\mathbf{u}-\mathbf{u}_{k+1}\right\| \leq\left[1-\kappa(\mathbf{A})^{-1}\left(\frac{\alpha-\omega}{1+\omega}\right)^{2}\right]^{\frac{1}{2}}\left\|\mathbf{u}-\mathbf{w}_{k}\right\|$.

Our $(k+1)$ th iterand is, however, not $\mathbf{u}_{k+1}$ but $\mathbf{w}_{k+1}$, which contains errors because of the nonexact right-hand side and the inexact solution of the Galerkin system. One can simply estimate $\left\|\mathbf{u}-\mathbf{w}_{k+1}\right\| \leq\left\|\mathbf{u}-\mathbf{u}_{k+1}\right\|+\left\|\mathbf{u}_{k+1}-\mathbf{w}_{k+1}\right\|$, but a sharper result can be derived by using that $\mathbf{u}-\mathbf{w}_{k+1}$ is nearly $\langle\langle\cdot, \cdot\rangle\rangle$-orthogonal 
to $\ell_{2}\left(\Lambda_{k+1}\right)$. With $\beta:=\gamma \frac{2+2 \omega}{\alpha-\omega} \kappa(\mathbf{A})^{\frac{1}{2}}<\frac{1}{3}$, we have

$$
\begin{aligned}
\left\|\mathbf{u}_{k+1}-\mathbf{w}_{k+1}\right\| & \leq\left\|\mathbf{A}^{-1}\right\|^{\frac{1}{2}}\left\|\mathbf{P}_{k+1} \mathbf{A}\left(\mathbf{u}_{k+1}-\mathbf{w}_{k+1}\right)\right\| \\
& \leq\left\|\mathbf{A}^{-1}\right\|^{\frac{1}{2}}\left(\left\|\mathbf{g}_{k+1}-\mathbf{P}_{k+1} \mathbf{A} \mathbf{w}_{k+1}\right\|+\left\|\mathbf{P}_{k+1} \mathbf{f}-\mathbf{g}_{k+1}\right\|\right) \\
& \leq\left\|\mathbf{A}^{-1}\right\|^{\frac{1}{2}} 2 \gamma \nu_{k} \leq\left\|\mathbf{A}^{-1}\right\|^{\frac{1}{2}} 2 \gamma \frac{1+\omega}{\alpha-\omega}\left\|\mathbf{P}_{k+1}\left(\mathbf{f}-\mathbf{A} \mathbf{w}_{k}\right)\right\| \\
& \leq \beta\left\|\mathbf{u}_{k+1}-\mathbf{w}_{k}\right\| .
\end{aligned}
$$

Using $\mathbf{u}-\mathbf{u}_{k+1} \perp_{\langle\langle,\rangle} \ell_{2}\left(\Lambda_{k+1}\right)$, we have

$$
\begin{aligned}
\left|\left\langle\left\langle\mathbf{u}-\mathbf{w}_{k+1}, \mathbf{w}_{k+1}-\mathbf{w}_{k}\right\rangle\right\rangle\right| & =\left|\left\langle\left\langle\mathbf{u}_{k+1}-\mathbf{w}_{k+1}, \mathbf{w}_{k+1}-\mathbf{w}_{k}\right\rangle\right\rangle\right| \\
& \leq\left\|\mathbf{u}_{k+1}-\mathbf{w}_{k+1}\right\|\|\| \mathbf{w}_{k+1}-\mathbf{w}_{k} \| \\
& \leq \beta\left\|\mathbf{u}_{k+1}-\mathbf{w}_{k}\right\|\|\| \mathbf{w}_{k+1}-\mathbf{w}_{k} \| .
\end{aligned}
$$

Now by writing

$$
\left\|\mathbf{u}-\mathbf{w}_{k}\right\|^{2}=\left\|\mathbf{u}-\mathbf{w}_{k+1}\right\|^{2}+\left\|\mathbf{w}_{k+1}-\mathbf{w}_{k}\right\|^{2}+2\left\langle\left\langle\mathbf{u}-\mathbf{w}_{k+1}, \mathbf{w}_{k+1}-\mathbf{w}_{k}\right\rangle\right\rangle
$$

and, for obtaining the second line in the following multiline formula, two applications of

$$
\left\|\mathbf{w}_{k+1}-\mathbf{w}_{k}\right\| \geq\left\|\mathbf{u}_{k+1}-\mathbf{w}_{k}\right\|-\left\|\mathbf{w}_{k+1}-\mathbf{u}_{k+1}\right\| \geq(1-\beta)\left\|\mathbf{u}_{k+1}-\mathbf{w}_{k}\right\|,
$$

we find that

$$
\begin{aligned}
\left\|\mathbf{u}-\mathbf{w}_{k}\right\|^{2} & \geq\left\|\mathbf{u}-\mathbf{w}_{k+1}\right\|^{2}+\left\|\mathbf{w}_{k+1}-\mathbf{w}_{k}\right\|\left(\left\|\mathbf{w}_{k+1}-\mathbf{w}_{k}\right\|-2 \beta\left\|\mathbf{u}_{k+1}-\mathbf{w}_{k}\right\|\right) \\
& \geq\left\|\mathbf{u}-\mathbf{w}_{k+1}\right\|^{2}+(1-\beta)(1-3 \beta)\left\|\mathbf{u}_{k+1}-\mathbf{w}_{k}\right\|^{2} \\
& \geq\left\|\mathbf{u}-\mathbf{w}_{k+1}\right\|^{2}+(1-\beta)(1-3 \beta) \kappa(\mathbf{A})^{-1}\left(\frac{\alpha-\omega}{1+\omega}\right)^{2}\left\|\mathbf{u}-\mathbf{w}_{k}\right\|^{2}
\end{aligned}
$$

or

$$
\left\|\mathbf{u}-\mathbf{w}_{k+1}\right\| \leq\left[1-(1-\beta)(1-3 \beta) \kappa(\mathbf{A})^{-1}\left(\frac{\alpha-\omega}{1+\omega}\right)^{2}\right]^{\frac{1}{2}}\left\|\mathbf{u}-\mathbf{w}_{k}\right\|,
$$

which completes the proof.

Remark 2.8. Inside the call of $\left[\Lambda_{k+1}, \nu_{k}\right]=\mathbf{G R O W}\left[\mathbf{w}_{k}, \theta \nu_{k-1}, \varepsilon\right]$ made in SOLVE, we search an approximation $\mathbf{r}_{k, \zeta}:=\mathbf{R H S}[\zeta / 2]-\mathbf{A P P L Y}\left[\mathbf{w}_{k}, \zeta / 2\right]$ for $\overline{\mathbf{r}}_{k}:=\mathbf{f}-$ $\mathbf{A} \mathbf{w}_{k}$ with a $\zeta \leq \omega\left\|\mathbf{r}_{k, \zeta}\right\|$ that is as large as possible in order to minimize the support of $\mathbf{r}_{k, \zeta}$ outside supp $\mathbf{w}_{k}$. When $k>0$, because of the preceding calls of RHS and GALSOLVE, we have a set $\Lambda_{k} \supset \operatorname{supp} \mathbf{w}_{k}$ and a $\nu_{k-1}$ with $\left\|\mathbf{P}_{\Lambda_{k}} \overline{\mathbf{r}}_{k}\right\| \leq$ $\delta_{k}:=2 \gamma \nu_{k-1}$. In this remark, we investigate whether it is possible to benefit from this information to obtain an approximation for the residual with relative error not exceeding $\omega$ whose support extends less outside $\operatorname{supp} \mathbf{w}_{k}$.

Let $\mathbf{r}_{k, \zeta}^{I}:=\mathbf{P}_{\Lambda_{k}} \mathbf{r}_{k, \zeta}$ and $\mathbf{r}_{k, \zeta}^{E}:=\mathbf{P}_{\nabla \backslash \Lambda_{k}} \mathbf{r}_{k, \zeta}$, and similarly $\overline{\mathbf{r}}_{k}^{I}$ and $\overline{\mathbf{r}}_{k}^{E}$. From

$$
\zeta^{2} \geq\left\|\overline{\mathbf{r}}-\mathbf{r}_{k, \zeta}\right\|^{2}=\left\|\overline{\mathbf{r}}^{I}-\mathbf{r}_{k, \zeta}^{I}\right\|^{2}+\left\|\overline{\mathbf{r}}^{E}-\mathbf{r}_{k, \zeta}^{E}\right\|^{2} \geq\left(\left\|\mathbf{r}_{k, \zeta}^{I}\right\|-\delta_{k}\right)^{2}+\left\|\overline{\mathbf{r}}^{E}-\mathbf{r}_{k, \zeta}^{E}\right\|^{2},
$$

we have

$$
\left\|\overline{\mathbf{r}}-\mathbf{r}_{k, \zeta}^{E}\right\|=\left(\left\|\overline{\mathbf{r}}^{E}-\mathbf{r}_{k, \zeta}^{E}\right\|^{2}+\left\|\overline{\mathbf{r}}^{I}\right\|^{2}\right)^{\frac{1}{2}} \leq\left(\zeta^{2}-\left(\left\|\mathbf{r}_{k, \zeta}^{I}\right\|-\delta_{k}\right)^{2}+\delta_{k}^{2}\right)^{\frac{1}{2}}=: \breve{\zeta} .
$$

So, alternatively, instead of $\mathbf{r}_{k, \zeta}$, we may use $\mathbf{r}_{k, \zeta}^{E}$ as an approximation for $\overline{\mathbf{r}}_{k}$, and thus stop the routine GROW as soon as $\nu_{k}:=\left\|\mathbf{r}_{k, \zeta}^{E}\right\|+\breve{\zeta} \leq \varepsilon$ or $\breve{\zeta} \leq \omega\left\|\mathbf{r}_{k, \zeta}^{E}\right\|$, and use $\mathbf{r}_{k, \zeta}^{E}$ also for the determination of $\Lambda_{k+1}$. Since for any $\zeta$ and $\mathbf{r}_{k, \zeta}$ with $\mathbf{r}_{k, \zeta}^{I} \neq 0$ and $\zeta<\left\|\mathbf{r}_{k, \zeta}\right\|$, it holds that $\breve{\zeta}\left\|\mathbf{r}_{k, \zeta}\right\|<\zeta\left\|\mathbf{r}_{k, \zeta}^{E}\right\|$ if $\delta_{k}$ is small enough, under this condition the alternative test is passed more easily. This may even be a reason to decrease the parameter $\gamma$. 
The approach discussed in this remark has been applied in the experiments reported in [DHS05].

\section{NUMERICAL EXPERIMENT}

We consider the variational formulation of the following problem of order $2 t=2$ on the interval $[0,1]$, i.e., $n=1$, with periodic boundary conditions

$$
-\Delta u+u=f \quad \text { on } \mathbb{R} / \mathbb{Z} \text {. }
$$

We define the right-hand side $f$ by $f(v)=4 v\left(\frac{1}{2}\right)+\int_{0}^{1} g(x) v(x) d x$, with

$$
g(x)=\left(16 \pi^{2}+1\right) \cos (4 \pi x)-4+ \begin{cases}2 x^{2}, & \text { if } x \in[0,1 / 2), \\ 2(1-x)^{2}, & \text { if } x \in[1 / 2,1],\end{cases}
$$

so that the solution $u$ is given by

$$
u(x)=\cos (4 \pi x)+ \begin{cases}2 x^{2}, & \text { if } x \in[0,1 / 2), \\ 2(1-x)^{2}, & \text { if } x \in[1 / 2,1]\end{cases}
$$

(see Figure 1).

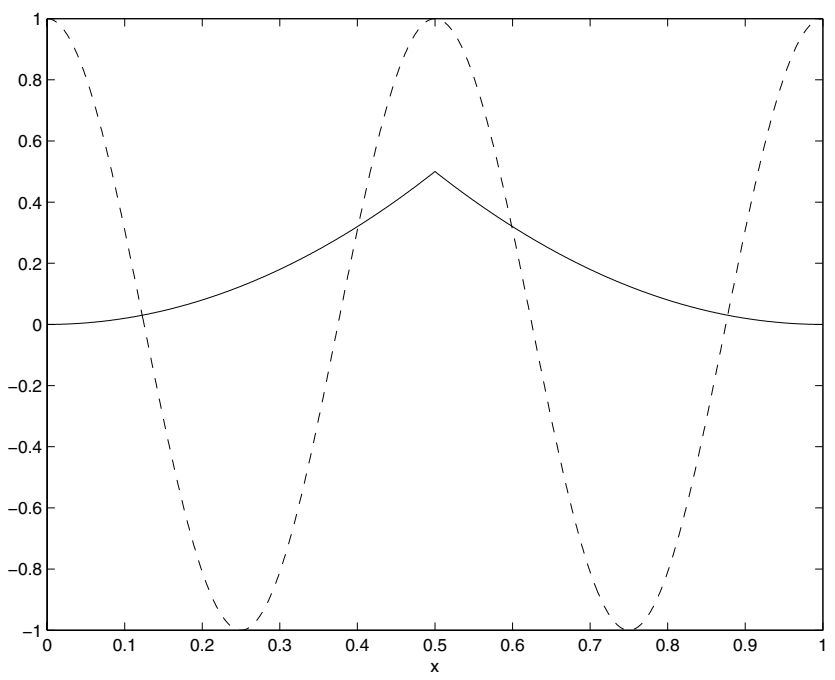

Figure 1. The solution $u$ is the sum of both functions illustrated.

We use the periodized B-spline wavelets of order $d=3$ with $\tilde{d}=3$ vanishing moments from CDF92 normalized in the $H^{1}(0,1)$-norm. The solution $u$ is in $H^{s+1}(\mathbb{R} / \mathbb{Z})$ only for $s<\frac{1}{2}$. On the other hand, since $u$ can be shown to be in $B_{\tau}^{s+1}\left(L_{\tau}(\mathbb{R} / \mathbb{Z})\right)$ for any $s>0$, we deduce that the corresponding discrete solution $\mathbf{u}$ is in $\ell_{\tau}^{w}$ for any $s<\frac{d-t}{n}=2$.

Each entry of the infinite stiffness matrix A can be computed in $\mathcal{O}(1)$ operations. By applying the compression rules from [Ste04, we see that $\mathbf{A}$ is $s^{*}$-computable with $s^{*}=t+\tilde{d}=4$.

For developing a routine RHS, we split $f=f_{1}+f_{2}$, where $f_{1}(v)=\int_{0}^{1} f_{1}(x) v(x) d x$ with $f_{1}(x)=\left(16 \pi^{2}+1\right) \cos (4 \pi x)-4$. Correspondingly, we split $\mathbf{f}=\mathbf{f}_{1}+\mathbf{f}_{2}$ and, given a tolerance $\varepsilon$, we approximate both infinite vectors within tolerance $\varepsilon / 2$ by, 
for suitable $\ell_{1}(\varepsilon), \ell_{2}(\varepsilon)$, dropping all coefficients with indices $\lambda$ with $|\lambda|>\ell_{1}(\varepsilon)$ or $|\lambda|>\ell_{2}(\varepsilon)$, respectively.

From

$$
\left|\left\langle\psi_{\lambda}, f_{1}\right\rangle\right| \leq\left\|\psi_{\lambda}\right\|_{L_{1}(0,1)} \inf _{p \in P_{2}}\left\|f_{1}-p\right\|_{L_{\infty}\left(\operatorname{supp} \psi_{\lambda}\right)},
$$

$\inf _{p \in P_{2}}\left\|f_{1}-p\right\|_{L_{\infty}\left(\operatorname{supp} \psi_{\lambda}\right)} \leq\left(\frac{\pi}{4} \operatorname{diam}\left(\operatorname{supp} \psi_{\lambda}\right)\right)^{3}\left\|f_{1}^{\prime \prime \prime}\right\|_{L_{\infty}(0,1)} / 3$ ! (Jackson estimate) $\left.\left\|\psi_{\lambda}\right\|_{L_{1}(0,1)} \leq \operatorname{diam}\left(\operatorname{supp} \psi_{\lambda}\right)\right)^{\frac{1}{2}}\left\|\psi_{\lambda}\right\|_{L_{2}(0,1)}, \operatorname{diam}\left(\operatorname{supp} \psi_{\lambda}\right)=5 \cdot 2^{-|\lambda|}$, and, with $\psi$ being the "mother wavelet", $\left\|\psi_{\lambda}\right\|_{L_{2}(0,1)}=\left[4^{|\lambda|}\left\|\psi^{\prime}\right\|_{L_{2}(\mathbb{R})}^{2} /\|\psi\|_{L_{2}(\mathbb{R})}^{2}+1\right]^{-1}$, and $\#\{\lambda:|\lambda|=k\}=2^{k}$, we find an upper bound for the error $\sqrt{\sum_{|\lambda|>\ell_{1}(\varepsilon)}\left|\left\langle\psi_{\lambda}, f_{1}\right\rangle\right|^{2}}$ which is $\approx 2^{-4 \ell_{1}(\varepsilon)}$. Setting this upper bound equal to $\varepsilon / 2$ and solving for $\ell_{1}(\varepsilon)$ gives an approximation for $\mathbf{f}_{1}$ of length $\sim 2^{\ell_{1}(\varepsilon)} \approx \varepsilon^{-1 / 4}$. Note that in view of the assumption we made on RHS, a vector length $\approx \varepsilon^{-1 / 2}$ would have been sufficient. Such a length would have been found with wavelets that have 1 vanishing moment.

From

$$
\left|\left\langle\psi_{\lambda}, f_{2}\right\rangle\right| \leq\left(4+\left\|g-f_{1}\right\|_{L_{1}\left(\operatorname{supp} \psi_{\lambda}\right)}\right)\left\|\psi_{\lambda}\right\|_{L_{\infty}(0,1)},
$$

$\left\|g-f_{1}\right\|_{L_{1}\left(\operatorname{supp} \psi_{\lambda}\right)} \leq \frac{1}{2} 5 \cdot 2^{-|\lambda|},\left\|\psi_{\lambda}\right\|_{L_{\infty}(0,1)}=\left[2^{|\lambda| / 2}\|\psi\|_{L_{\infty}(\mathbb{R})} /\|\psi\|_{L_{2}(\mathbb{R})}\right]\left\|\psi_{\lambda}\right\|_{L_{2}(0,1)}$, $\#\left\{|\lambda|=k: \frac{1}{2}\right.$ is an interior point of $\left.\operatorname{supp} \psi_{\lambda}\right\}=9$, and the fact that $\left\langle\psi_{\lambda}, f_{2}\right\rangle$ vanishes when $\lambda$ is not in any of these sets, we find an upper bound for the error $\sqrt{\sum_{|\lambda|>\ell_{2}(\varepsilon)}\left|\left\langle\psi_{\lambda}, f_{2}\right\rangle\right|^{2}}$ which is $\approx 2^{-\ell_{2}(\varepsilon) / 2}$. Setting this upper bound equal to $\varepsilon / 2$ and solving for $\ell_{2}(\varepsilon)$ gives an approximation for $\mathbf{f}_{2}$ of length $\leq 9\left(\ell_{2}(\varepsilon)+1\right)=$ $\mathcal{O}(|\log (\varepsilon)|+1)$, which is asymptotically even much smaller than the bound we found in the $\mathbf{f}_{1}$ case.

We will compare the results of our adaptive wavelet algorithm SOLVE with those obtained with the Richardson iteration based method from [CDD02, which we refer to as being the CDD2 method, and that reads as follows:

CDD2SOLVE $[\nu, \varepsilon] \rightarrow \mathbf{w}:$

$\% \nu \geq\|\mathbf{u}\|$

$\%$ Define the parameters $\omega:=\frac{2}{\|\mathbf{A}\|+\left\|\mathbf{A}^{-1}\right\|^{-1}}$ and $\rho:=\frac{1-\kappa(\mathbf{A})}{1+\kappa(\mathbf{A})}$.

$\%$ Let $\theta$ and $K$ be constants with $2 \rho^{K}<\theta<1 / 2$.

$\mathbf{w}:=0$

while $\nu>\varepsilon$ do

$$
\begin{aligned}
& \text { for } j=1 \text { to } K \\
& \quad \mathbf{w}:=\mathbf{w}+\omega\left(\mathbf{R H S}\left[\frac{\rho^{j} \nu}{2 \omega K}\right]-\operatorname{APPLY}\left[\mathbf{w}, \frac{\rho^{j} \nu}{2 \omega K}\right]\right) \\
& \text { endfor } \\
& \nu:=2 \rho^{K} \nu / \theta \\
& \mathbf{w}:=\operatorname{COARSE}[\mathbf{w},(1-\theta) \nu]
\end{aligned}
$$

enddo

Here the coarsening routine COARSE is defined by

COARSE $[\mathbf{w}, \delta] \rightarrow \mathbf{w}_{\delta}$ with $\left\|\mathbf{w}_{\delta}-\mathbf{w}\right\| \leq \delta$, where modulo some absolute factor \#supp $\mathbf{w}_{\delta}$ is minimal.

We tested our adaptive wavelet algorithm SOLVE or CDD2SOLVE with parameters $\alpha=0.4, \omega=0.012618$, and $\gamma=0.009581$, or $K=5$ and $\theta=2 / 7$, respectively. Inside the ranges where the methods are proven to be of optimal computational complexity, these parameters are close to the values that give quantitatively the best results. Actually, since these ranges result from a succession of 
worst case analyses, we may expect that outside them, i.e., concerning SOLVE for larger $\alpha, \omega$ and $\gamma$, more efficient algorithms are obtained. The numerical results, given in Figure 2, illustrate the optimal computational complexity of both SOLVE and CDD2SOLVE. Note that the time measurements do not start at zero, but after $10^{0}=1$ second. The results show that in this example the new method needs less than a factor 10 in computing time to achieve the same accuracy.

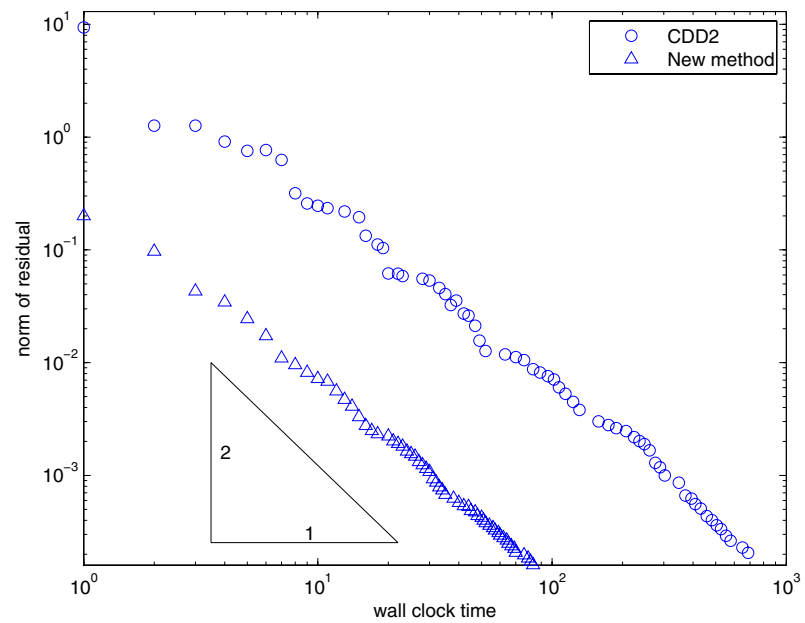

Figure 2. Convergence histories

\section{REFERENCES}

[CDD01] A. Cohen, W. Dahmen, and R. DeVore. Adaptive wavelet methods for elliptic operator equations - Convergence rates. Math. Comp, 70:27-75, 2001. MR1803124(2002h:65201)

[CDD02] A. Cohen, W. Dahmen, and R. DeVore. Adaptive wavelet methods II - Beyond the elliptic case. Found. Comput. Math., 2(3):203-245, 2002. MR1907380 (2003f:65212)

[CDF92] A. Cohen, I. Daubechies, and J.C. Feauveau. Biorthogonal bases of compactly supported wavelets. Comm. Pur. Appl. Math., 45:485-560, 1992. MR1162365 (93e:42044)

[CM00] A. Cohen and R. Masson. Wavelet adaptive method for second order elliptic problems: Boundary conditions and domain decomposition. Numer. Math., 86:193-238, 2000. MR.1777487 (2001j:65185)

[Coh03] A. Cohen. Numerical Analysis of Wavelet Methods. Elsevier, Amsterdam, 2003. MR.1990555 (2004c:65178)

[CTU99] C. Canuto, A. Tabacco, and K. Urban. The wavelet element method part I: Construction and analysis. Appl. Comput. Harmon. Anal., 6:1-52, 1999. MR1664902 (99k:42055)

[Dah99] S. Dahlke. Besov regularity for elliptic boundary value problems in polygonal domains. Appl. Math. Lett., 12(6):31-36, 1999. MR1751404 (2001b:35077)

[DD97] S. Dahlke and R. DeVore. Besov regularity for elliptic boundary value problems. Comm. Partial Differential Equations, 22(1 \& 2):1-16, 1997. MR.1434135 (97k:35047)

[DeV98] R. DeVore. Nonlinear approximation. Acta Numer., 7:51-150, $1998 . \quad$ MR 1689432 (2001a:41034)

[DFR04] S. Dahlke, M. Fornasier, and T. Raasch. Adaptive frame methods for elliptic operator equations. Bericht Nr. 2004-3, Philipps-Universität Marburg, 2004. To appear in Adv. Comput. Math. 
[DHS05] W. Dahmen, H. Harbrecht, and R. Schneider. Adaptive methods for boundary integral equations - complexity and convergence estimates. IGPM report 250, RWTH Aachen, March 2005. To appear in Math. Comp.

[DS99a] W. Dahmen and R. Schneider. Composite wavelet bases for operator equations. Math. Comp., 68:1533-1567, 1999. MR.1648379(99m:65122)

[DS99b] W. Dahmen and R. Schneider. Wavelets on manifolds I: Construction and domain decomposition. SIAM J. Math. Anal., 31:184-230, 1999. MR.1742299 (2000k:65242)

[GS06a] T. Gantumur and R.P. Stevenson. Computation of differential operators in wavelet coordinates. Math. Comp. 75:697-709, 2006. MR2196987

[GS06b] T. Gantumur and R.P. Stevenson. Computation of singular integral operators in wavelet coordinates. Computing, 76:77-107, 2006. MR2174673 (2006e:65051)

[HS06] H. Harbrecht and R.P. Stevenson. Wavelets with patchwise cancellation properties. Math. Comp., 75(256):1871-1889, 2006.

[Ste03] R.P. Stevenson. Adaptive solution of operator equations using wavelet frames. SIAM J. Numer. Anal., 41(3):1074-1100, 2003. MR2005196 (2004e:42062)

[Ste06] R.P. Stevenson. Composite wavelet bases with extended stability and cancellation properties. Technical Report 1345, Department of Mathematics, Utrecht University, January 2006. To appear in SIAM J. Numer. Anal.

[Ste04] R.P. Stevenson. On the compressibility of operators in wavelet coordinates. SIAM J. Math. Anal., 35(5):1110-1132, 2004. MR2050194 (2005e:42128)

[vS04] J. van den Eshof and G.L.G. Sleijpen. Inexact Krylov subspace methods for linear systems. SIAM J. Matrix Anal. Appl., 26(1):125-153, 2004. MR2106939 (2005i:65046)

Department of Mathematics, Utrecht University, P.O. Box 80.010, NL-3508 TA Utrecht, The Netherlands

E-mail address: gantumur@math.uu.nl

URL: http://www.math.uu.nl/people/gantumur/

Institute of Computer Science and Applied Mathematics, Christian-Albrechts-UniVersity of Kiel, Olshausenstr. 40, 24098 Kiel, Germany

E-mail address: hh@numerik.uni-kiel.de

$U R L:$ http://www. numerik. uni-kiel.de/ hh/

Department of Mathematics, Utrecht University, P.O. Box 80.010, NL-3508 TA Utrecht, The NetherLands

E-mail address: stevenson@math.uu.nl

URL: http://www.math.uu.nl/people/stevenso/ 